\title{
Self-Assembled Conductive Network of Carbon Nanotubes-Polyaniline as Potential Nanocomposites
}

\author{
S. Gupta ${ }^{*}, 1$ and Y. Ding ${ }^{2, \S}$ \\ ${ }^{I}$ Department of Electrical and Computer Engineering, University of Missouri-Columbia, MO 65211, USA \\ ${ }^{2}$ Crosslink Polymer Research, St. Louis, MO 63026, USA
}

\begin{abstract}
Carbon nanotubes are of great interest because of unique physical (mechanical, electrical, thermal, and chemical) properties. Especially their large elastic modulus and breaking strength make them highly attractive for their use as reinforced agents for traditional as well as conductive polymers forming a new class of multifunctional advanced carbon composites-nanocomposites. This is in addition to high electrical conductivity achieved through lower percolation thresholds for multitude of applications. Polyaniline (PANI) has a high potential due to its ease of synthesis, excellent environmental and thermal stability and reversible control of its electrical properties. A variant of PANI doped with DNNSA (dinonyl napththalene sulfonic acid) facilitates more promise by making it soluble and easily processable. In this work, DNNSA-PANI is used as a matrix for both the single- and multiwalled carbon nanotubes as nanoscale reinforced agents. The films were prepared with varying nanotube contents synthesized by spin-cast preceded by ultrasonic mixing of the constituents for a few hours. They were characterized using complementary analytical tools include scanning electron microscopy, atomic force microscopy, X-Ray diffraction, visible micro-Raman spectroscopy and room temperature $d c$ electrical conductivity. These techniques reveal their morphology and microscopic structure and physical properties that help to establish process-microstructure-property relationship. The resulting nanocomposites possess enhanced or new sets of physical properties. However, because of occasional presence of inhomogeneities, the interfacial interactions and physical properties are 'site-selective' revealed using Raman spectroscopy ascribed to the charge transfer. The present work also discusses some of the findings in light of self-alignment of nanotubes in polymer matrix and their optical and electrical properties keeping in view of their applications ranging printable organic electronic and sensor devices, electrodes for fuel cell and high-energy density Li batteries, biosensing platform, space and naval uses.
\end{abstract}

\section{INTRODUCTION}

Carbon is a unique element that serves as a fertile playground for a variety of nanoscale structures with varying morphology, geometry and topology [1-3]. The discovery of carbon nanotubes (CNTs, here onwards) has generated enormous and sustained interest in nanoscience as well as nanotechnologies. CNTs can be divided into two main categories: single-walled carbon nanotubes (SWNT) and multiwalled carbon nanotubes (MWNT) depending upon the number of rolled up layers [4]. CNTs have shown exceptional electrical, mechanical and thermal properties, which are attractive for several potential applications ranging from nanoelectronics to mechanical and biomedical devices [5, 6]. While SWNT follow ballistic transport, these onedimensional quantum nanowires plays a significant role as interconnects in microelectronics and active components in optoelectronic nano-devices. In addition, their orientation with respect to substrate has an important impact on the performance of these devices. On the other hand, MWNT which are predominantly metallic character can be used for printable organic electronic devices and as novel platforms for nanomechanical devices [7, 8]. The exceptional Young's modulus of SWNT has prompted intensive studies of

*Address correspondence to this author at the Department of Electrical and Computer Engineering, University of Missouri-Columbia, MO 65211, USA; E-mail: sgup@ rocketmail.com; guptas@missouri.edu;

\$yiweiding@yahoo.com forming nanocomposites with polymers for printable organic electronics as well as space and naval applications $[9,10]$. However, the use of CNTs as reinforced agents in polymer matrix forming potential nanocomposites in practical applications has been largely limited by their relatively poor processability. Since they are practically insoluble and infusible, it leads to either aggregation or segregation thus resulting in nanoscale inhomogeneous samples [11]. Several groups across the world have attempted to mitigate this challenge by functionalizing these nanotubes using a variety of chemical groups, for instance [12-14].

As a result of the first report concerning the preparation of a CNTs/polymer composite [15] many efforts have been made to combine CNTs and polymers to produce functional nanocomposites with superior properties [16-20]. Apart from possible improvements in the mechanical and electrical properties of polymers, the formation of CNTs/polymer composites has been explored and still an ongoing effort for an effective incorporation of CNTs for the successful development of practical optical and electronic devices [21-23]. Composite materials based on the coupling of conducting organic polymers (COPs) and CNTs have shown that they possess properties of the individual components with a synergistic effect [24]. In this context, a special attention has been paid to the following COPs: polyaniline (PANI) [2527] polypyrrole (PPy) [28], poly[3-(2-hydroxyethyl)-2,5thienylene] (PHET) [7], polythiophene (PT) [29], poly (3,4ethylenedioxy thiophene) (PEDOT) [30] poly (p-phenylene 
vinylene) (PPV) [31] and poly (m-phenylene vinylene-co2,5-dioctoxy-p-phenylene) (PmPV) [32].

The combination of CNTs with COPs offers an attractive route to reinforce polymer matrix as well as to introduce novel physical properties based on morphological modification and/or electronic interaction between the individual components [21, 31]. Since doped conjugated polymers are the prototypical organic semiconductors, their choice as a starting point for conducting materials in organic electronics is apparent. Although when heavily doped they exhibit metallic state/phase with main drawbacks of insolubility and ease of oxidation $[33,34]$. Therefore the reinforced agent in nanocomposites not only enables easier processing and reduces component weight, but it also provides value-added physical properties including optical clarity, increased flame- resistance, resistance to oxidation and ablation and reduced gas permeability.

Among many COPs above mentioned, polyaniline (PANI) is unique because of its environmental and thermal stability and reversible electrical conductivity. The latter is controlled by well-known techniques of either chemical or electrochemical doping include the charge transfer reaction with the conjugated backbone and protonation of the nitrogen, respectively [35]. The conductivity of PANI is controlled by complete protonation with functionalized sulfonic acids [36]. Generally, the emeraldine base (EB) and emeraldine salt (ES, both polaronic and/or bipolaronic) forms of PANI can be interchanged by doping and de-doping with acid and base (Fig. 1) [37]. In this report, we present our recent results on the microscopic structure and physical

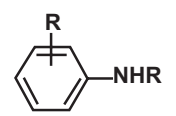

(a)

ANILINE MONOMER
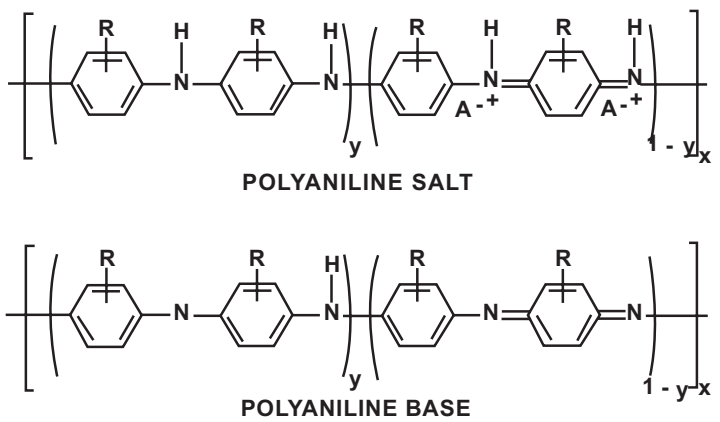

$\left(\left[\left(-\mathrm{C}_{6} \mathrm{H}_{4}-\mathrm{NH}-\mathrm{C}_{6} \mathrm{H}_{4}-\mathrm{NH}-\right)_{1-\mathrm{x}}\right]\left[\left(-\mathrm{C}_{6} \mathrm{H}_{4}-\mathrm{N}=\mathrm{C}_{6} \mathrm{H}_{4}=\mathrm{N}-\right)_{x}\right]\right)_{\mathrm{n}}$<smiles>CCCCCCCCCCCCCCCCCCCC</smiles>

$\left(\mathrm{C}_{9} \mathrm{H}_{19}\right)_{2} \mathrm{G}_{10} \mathrm{H}_{5} \mathrm{SO}_{3} \mathrm{H}$

(c)
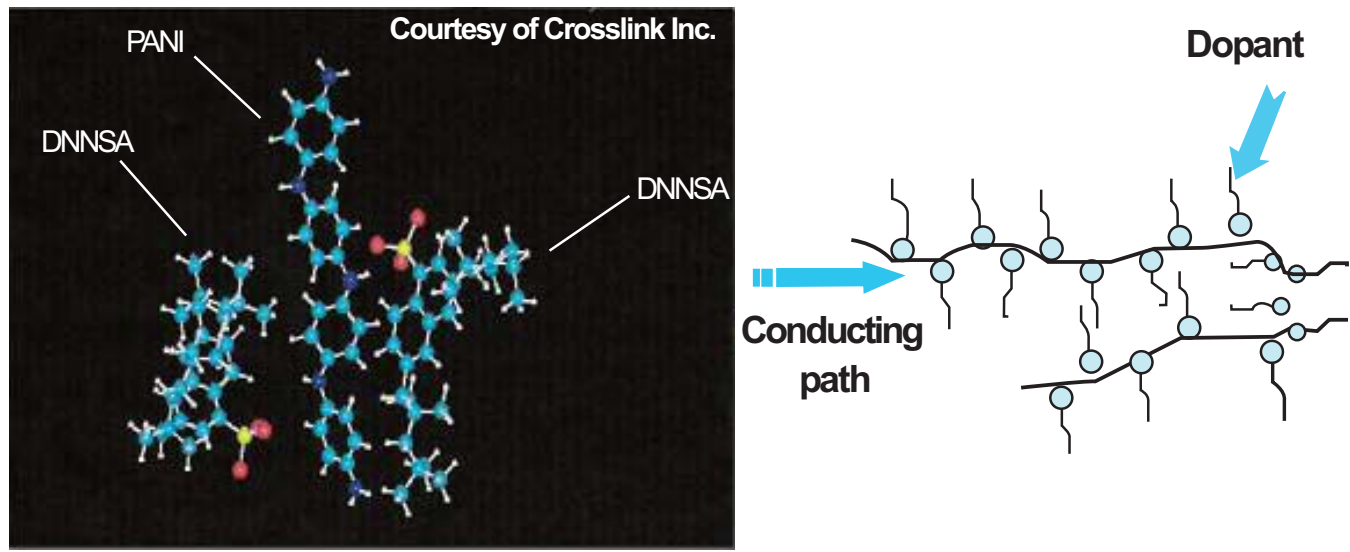

Fig. (1). (a) Shown are the chemical structure and the formula for PANI. Aniline monomer and polymer exist in a variety of molecular structures. R and R' represents hydrogen and/or alkyl or alkoxy functional groups containing between 1 and about 6 carbon atoms a piece. A represent an anion and $\mathrm{x}$ is an integer (b) molecular structure with molecular formula for DNNSA and (c) Schematic of doped PANI with DNNSA as a solubilizing side chain along the conducting path and (Courtesy of Crosslink Inc.). 
properties of DNNSA doped polyaniline-carbon nanotubes as self-assembled potential nanocomposites. In particular, we report some of the insights into the nanotube-polymer interface/interphase analyses using vibrational spectroscopy such as micro-Raman spectroscopy.

\section{EXPERIMENTAL DETAILS}

The polyaniline solution was prepared through emulsion polymerization following the procedure outlined in US Patent No. 5,863,465 with DNNSA dopant as a solubilizing side chain. Fig. (1) shows chemical structure and formula) [38]. Carbon Nanotubes Inc. (CNI) manufactured the SWNT used in the present study via a high-pressure fabrication method (HiPCO ${ }^{\circledR}$ SWNT) [39]. The MWNT were prepared using thermal chemical vapor deposition. We found that excellent dispersions of the nanotubes in DNNSA-PANI could be produced following two different procedures. The nanotubes could either be directly ultrasonicated into the DNNSAPANI solution or the dispersion of ultrasonicated nanotubes with xylene is sonicated with the DNNSA-PANI solution for a few hours. The results are reported for the former processing procedure for its ease and simplicity. Structural and physical property such as conductivity of the PANI-NT composites were measured by coating films of approximately $0.5 \mu \mathrm{m}$ thickness onto $\mathrm{Si}$ and glass slide with varying fractions of both the kinds of nanotubes (SWNT; 2.5 and 4.0wt. \% and MWNT; 0.25 and $0.50 \mathrm{wt} . \%$ ). Fig. (2) shows a flow diagram for preparation of nanocomposite films on a commercial Si. For surface morphological characterizations, we have used optical microscopy, scanning electron microscopy (JEOL Model 3400) and atomic force microscopy (AFM Veeco Instruments, Model Dimension 31). X-Ray diffraction measurements were performed using Siemens 5000 diffractometer to determine the lattice structure and spacing.

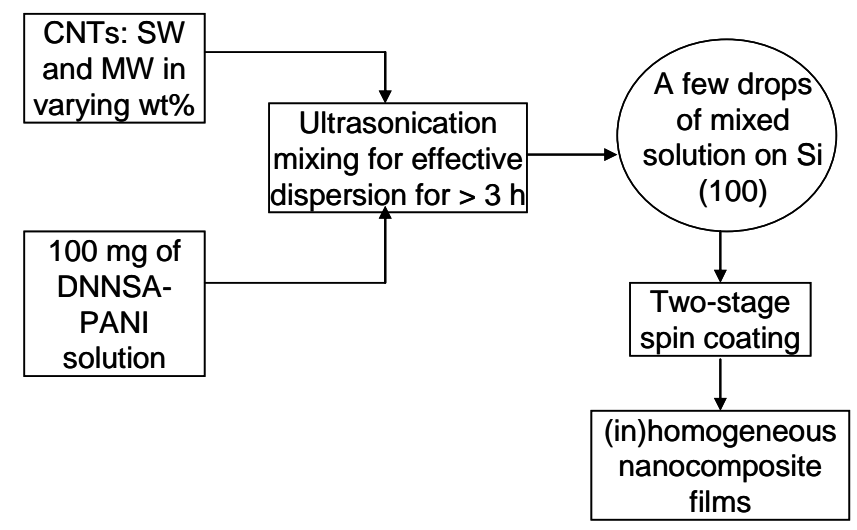

Fig. (2). Flow diagram of processing scheme for synthesizing conducting polymer-nanotube composite films.

We measured high-resolution Raman spectra with a typical 1.25-m, f/11 double monochromator, equipped with 2400 grating $\mathrm{g} / \mathrm{mm}$, ion-etched Super-Notch-Plus filter, $\mathrm{Ar}^{+}$laser operating at $\lambda_{\mathrm{L}}=514.5 \mathrm{~nm}\left(\mathrm{E}_{\mathrm{L}}=2.41 \mathrm{eV}\right)$ and at a power < $10 \mathrm{~mW}$ to avoid local laser heating with beam spot size about $<5$ micron, with a resolution of $\sim 2-4 \mathrm{~cm}^{-1}$. Data was acquired using Spectra Max software for Windows. All of the Raman spectra were recorded between a frequency range of $1000-3000 \mathrm{~cm}^{-1}$ in backscattered configuration and were analyzed using Jandel Scientific Peakfit software (v. 4.0) based on Marquardt-Levenberg method [40]. For room temperature $d c$ resistivity measurements, silver paint was used as ohmic contacts. The electrical properties including I-V and $d c$ electrical conductivity was measured using a standard two-probe and four-probe method.

\section{RESULTS AND DISCUSSION}

In Figs. (3) and (4), we display a series of optical microscopy, SEM and AFM images revealing the surface morphology of DNNSA-PANI/CNT composite films. The films show globular or cobbled stone-like morphology and spherical aggolmerates that becomes much apparent in $2 \mathrm{D}$ and $3 \mathrm{D}$ AFM images. The images also show that the films are relatively inhomogeneous and the nanotubes seem to appear poorly dispersed as anticipated. It requires further attention in terms of improving the processability of as-deposited albeit ultrasonicated nanotubes in conducting polymers. One of the approaches we adopted to enhance the solubility and/or dispersion of nanotubes is to functionalize them covalent or non-covalently. This approach seemed to improve the homogeneity and structural integrity. Fig. (5) shows possible arrangements of nanotubes incorporation in conducting polymer chains including self-assembled folded chain (Fig. 5a) and locally functionalized folded chain (Fig. 5b). From our microscopy images, it is apparent that the former applies to our nanocomposite samples.

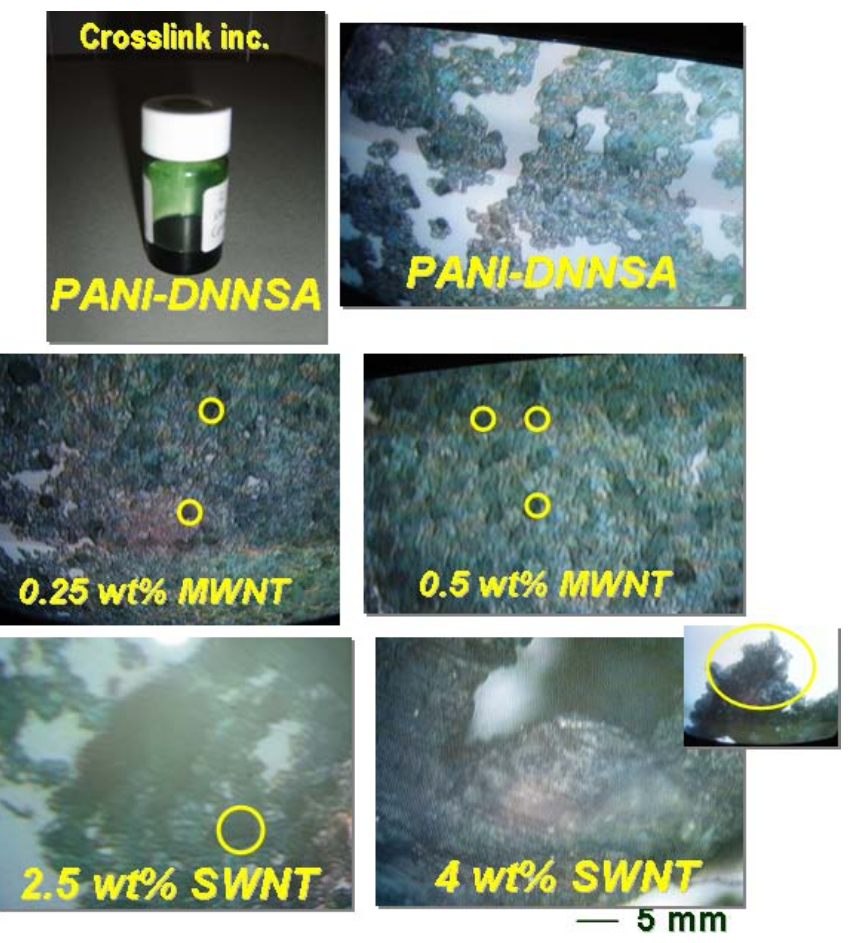

Fig. (3). Optical micrographs for DNNSA-PANI, MW and SW nanotubes incorporated films. The green solution in the vial is DNNSA-PANI.

$\mathrm{X}$-Ray diffraction (XRD) is used to determine the crystal structure of the solid materials. Fig. (6) shows XRD diffractograms for DNNSA-PANI and nanocomposites with varying nanotube concentration. Besides characteristic peaks at $27^{\circ}$ related to graphitic (002) plane and at $5^{\circ}$ relating (11) 

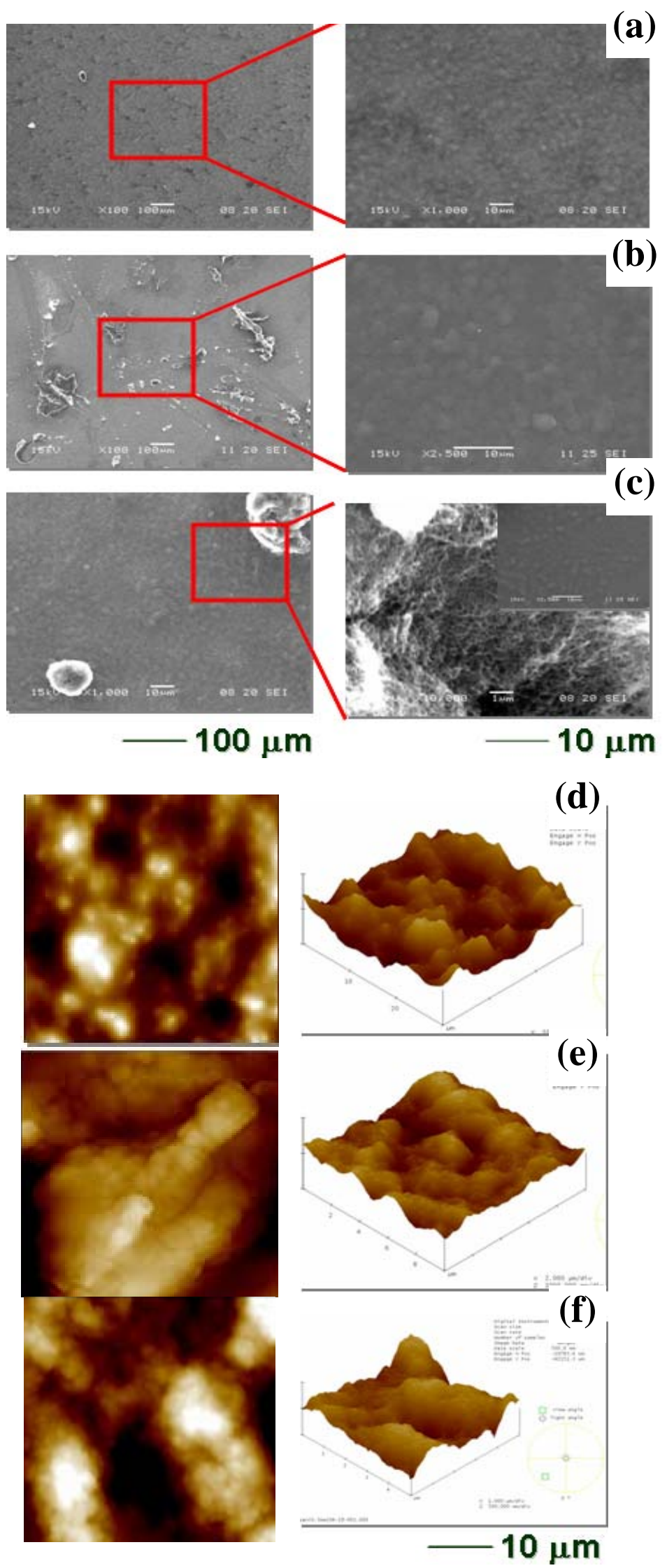

Fig. (4). (a-c) SEM images and (d-f) 2D and 3D AFM micrographs in $10 \mu \mathrm{m}$ x $10 \mu \mathrm{m}$ area showing the globular/spherical surface morphology for PANI-DNNSA, $0.25 \mathrm{wt} \% \mathrm{MW}$ and $2.5 \mathrm{wt} . \% \mathrm{SW}$ nanocomposite films. 

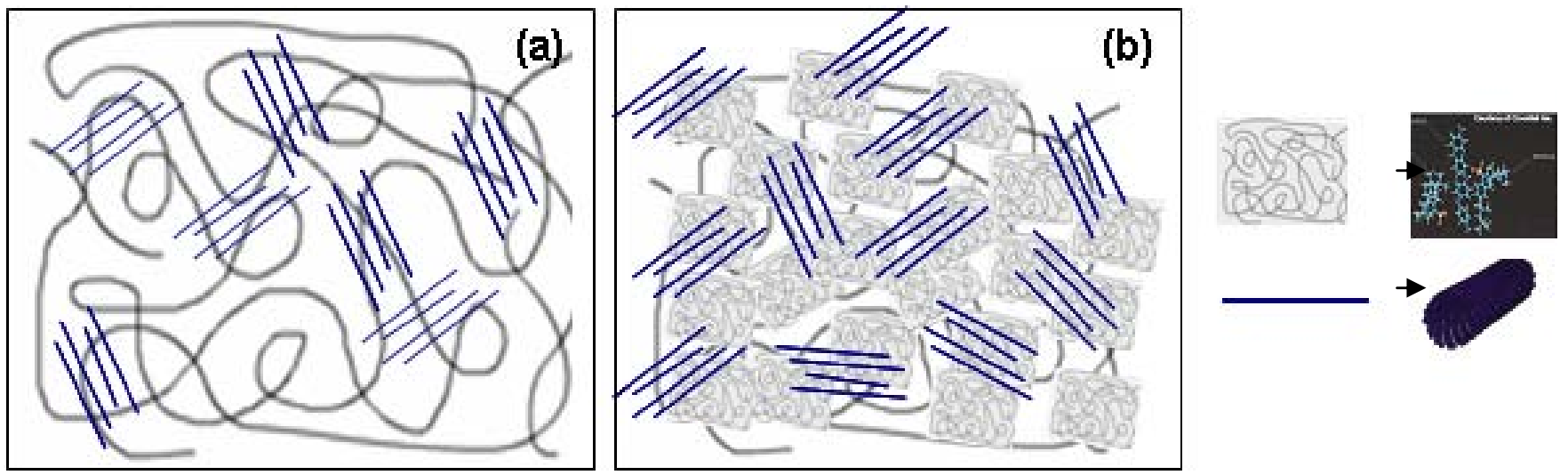

Fig. (5). Schematic of possible arrangements of nanotubes incorporation in conducting polymer chains forming potential nanocomposites (a) self-assembled in folded chain and (b) locally functionalized in folded chain. The symbols have their meaning as shown.

structure in SWNT (although weak), we also observe bands at $2 \theta=13.60^{\circ}$ and $15.0^{\circ}$ possibly related to DNNSA-PANI and composites. The position, width and shift of these bands are a direct measure of inter-planar spacing, crystallite size and stress/strain, respectively. Through careful fitting and further analyses using Bragg's law $2 d \operatorname{Sin} \theta=m \lambda$ and DebyeScherrer $L=\frac{K \lambda}{b \operatorname{Cos} \theta}$ formulae, where $\mathrm{K}($ Debye-Scherrer constant $)=0.94, \lambda=1.5405 \AA\left(\mathrm{Cu} \mathrm{K} \mathrm{K}_{\alpha}\right)$ and $\mathrm{b}$ is the fullwidth at half maximum of the peak, we found a systematic variation for the lattice spacing (d) and crystallite size (L) in the following order: $(\text { d) })_{\text {PANIISW }}<$ (d) $)_{\text {PANI/MW }}<$ (d) $<$ (d) PANI $_{\text {PAN }}$ and $(\mathrm{L})_{\text {PANIISW }}<(\mathrm{L})_{\text {PANI/MW }}<(\mathrm{L})_{\text {PANI }}$. In addition, the sharpness of these bands is reflective of high crystallinity, relatively high electrical conductivity and expanded coil of polymer matrix. However, whether it is caused by the presence of greater excess of DNNSA in the PANI and/or presence of nanotubes, is yet to be determined. Nevertheless, these observations demonstrate the importance of the hitherto unrecognized importance of the molecular conformations of a conducting polymer in conjunction with carbon nanotubes determining its electronic and electrical properties as well as morphology discussed below.

Raman spectroscopy (RS) has emerged as a powerful analytical tool for the structural characterization of carbonbased and other technologically important materials. RS is based on the inelastic light scattering that provides information about lattice vibrations (or phonons) of the probed material because of its sensitivity to changes in the atomic structure. The significance of RS experimental technique has increased substantially since it has proved helpful in understanding thus establishing the correlation between the vibrational properties and the microstructure of diverse nanoscale carbon materials [41]. The main reasons for this are the strong Raman response to the $\pi$-states due to resonance enhancement, its simplicity for the high-symmetry nanotubes, easy access and non-invasive nature.

Fig. (7) compares the first-order Raman spectra of representative multi-wall (MWNT) and single-wall nanotubes (SWNT) with different loading fractions of DNNSA-PANI. In the first-order, prominent Raman bands of interest in the
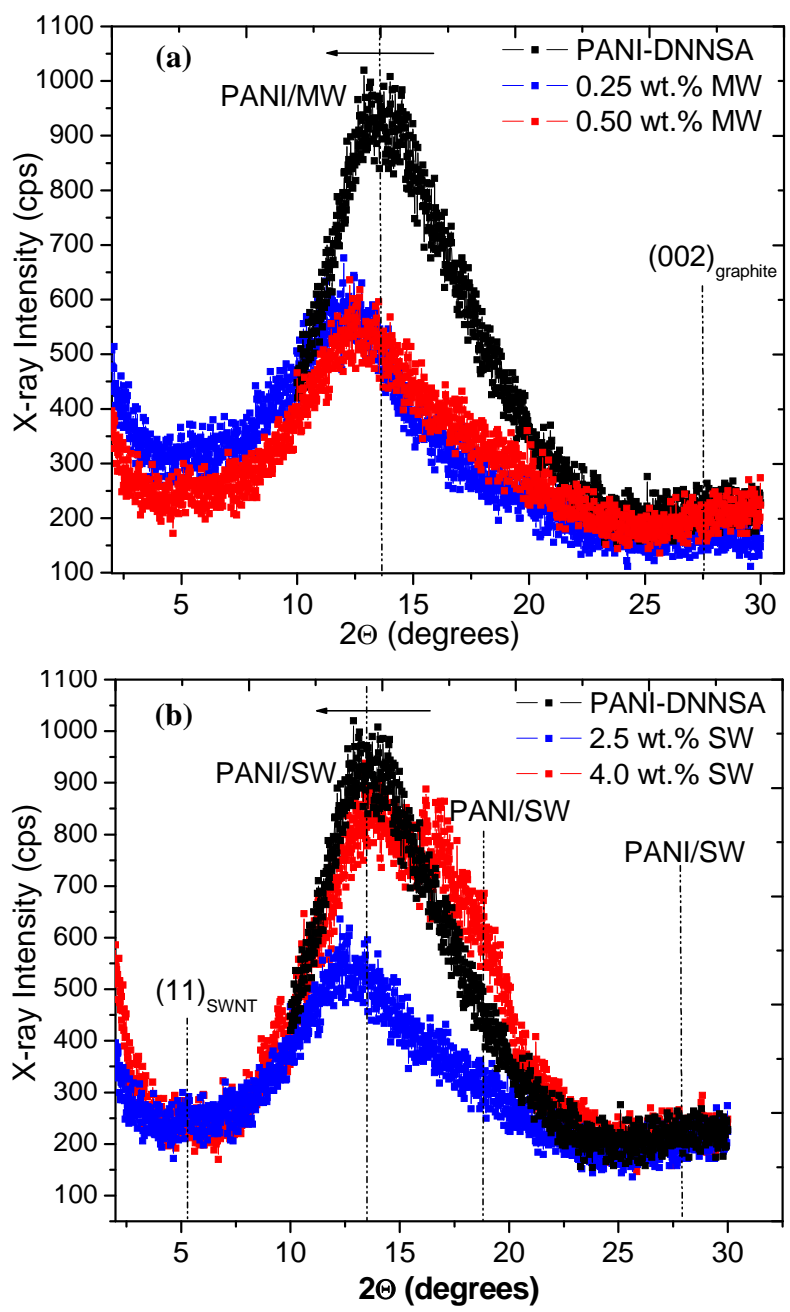

Fig. (6). X-Ray diffractograms showing the characteristic peaks and comparisons between the DNNSA-PANI/SW and DNNSAPANI/MW nanocomposites.

high frequency regime for all of the samples are $D$ and $G$ bands occurring at $\sim 1340 \mathrm{~cm}^{-1}\left(1350 \mathrm{~cm}^{-1}\right)$ and $\sim 1580 \mathrm{~cm}^{-1}$ $\left(1593 \mathrm{~cm}^{-1}\right)$, respectively [42]. For a realistic comparison the 

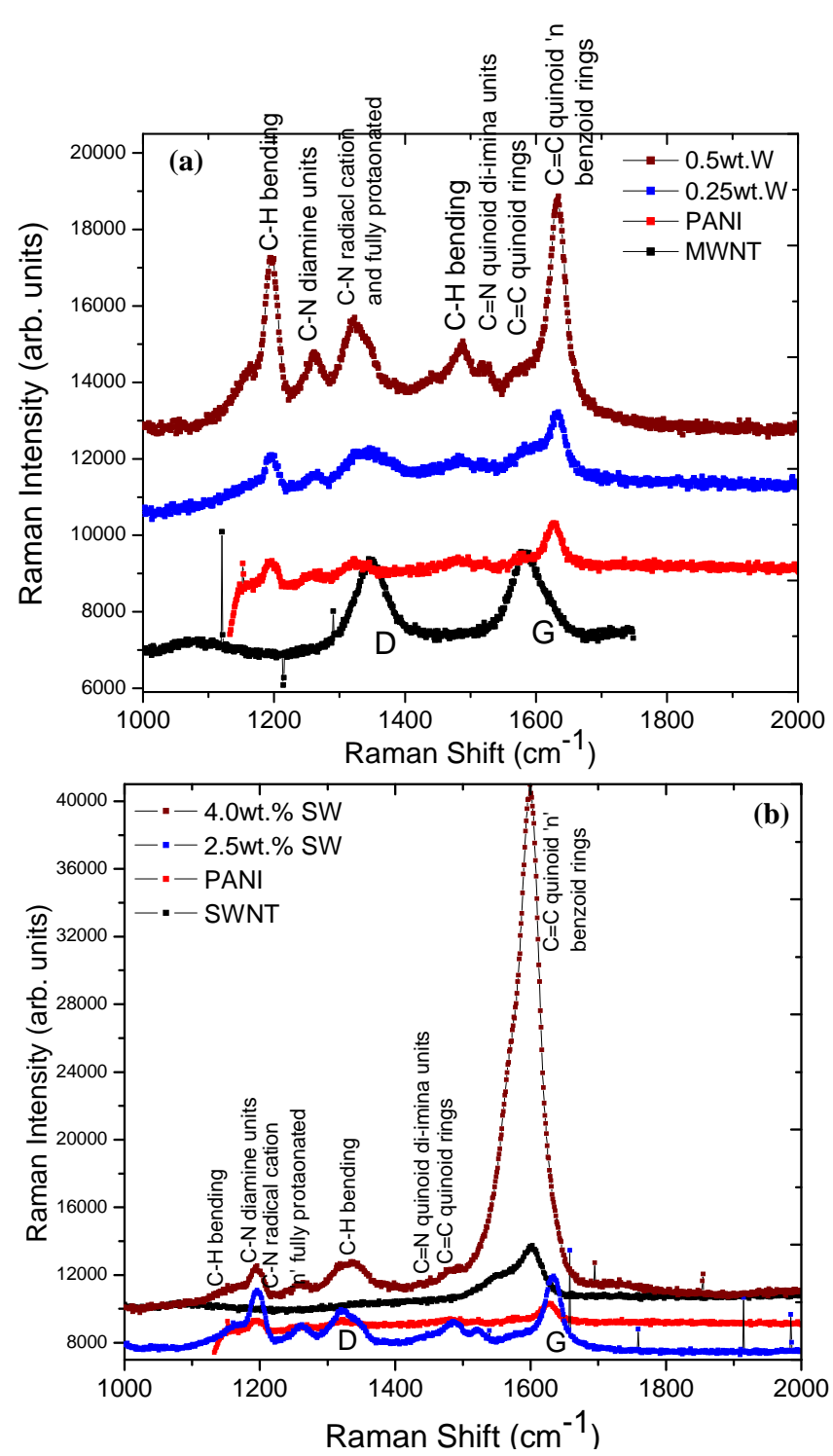

Fig. (7). Shown are the first-order visible micro-Raman spectra for all of the DNNSA-PANI-NT samples depicting the significant feature including $\mathrm{D}$ and $\mathrm{G}$ bands along with several others characteristic bands for DNNSA-PANI.

spectra were normalized with respect to the intense high frequency band (i.e. $\mathrm{G}$ band) and they are vertically shifted for clarity. The high-frequency band known as the tangential displacement mode (TDM) can be decomposed into two main peaks around $1562 \mathrm{~cm}^{-1}$ and $1593 \mathrm{~cm}^{-1}\left(\mathrm{~T}_{2}\right.$ and $\mathrm{T}_{3}$, respectively) with a shoulder at $\sim 1550 \mathrm{~cm}^{-1}\left(\mathrm{~T}_{1}\right)$ for SWNT (Fig. 7b). [Note that the $\mathrm{T}$ band is equivalent to the $\mathrm{G}$ band]. These features have been assigned to the $E_{2 g}$ symmetry mode of graphite that results from the splitting of intra-layer stretching mode. The latter arises due to a curvature induced re-hybridization $\left(\sigma^{*}-\pi^{*}\right)$ in smaller diameter nanotubes [43]. In fact, the re-hybridization of the $\sigma^{*}-\pi^{*}$ orbital strengthens the $\mathrm{C}-\mathrm{C}$ bond which yields relatively high elastic constants for the SWNT. The Raman modes for SWNT seem to be quite narrow and sharp indicating high uniformity, low level of impurities, smaller diameter distribution and primarily a semiconducting nature. This is in contrast to MWNT, which tend to be metallic and usually broader with substantial impurity [42]. The D peak of the $\mathrm{sp}^{2}$-bonded carbon materials is the disorder-activated band and it arises from the inplane substitutional hetero-atoms, vacancies, grain boundaries or other defects, all of which lower the translational symmetry and violate momentum conservation. Due to several concentric tubules in MWNT, this peak tends to gain intensity.

Excitation wavelength of $514.5 \mathrm{~nm}$ preferentially excites semiconducting tubes for SWNT and only fewer metallic and secondary tubes having smaller diameter such as in double-walled carbon nanotubes (DWNT). It is due to the resonant Raman scattering process via $\mathrm{E}_{22}{ }^{\mathrm{SS}}$ branch in van Hove singularities present in the electronic density of states (EDOS) for SWNT (Fig. 7b). The line broadening has been attributed to Fano resonance arising due to electron-phonon coupling (EPC) [44]. Generally, both the MWNT and SWNT exhibit a rather complicated spectral behavior depending on the loading in the COP, where a variety of covalent bonding may be formed. The presence of bands near 1500 and 1600 $\mathrm{cm}^{-1}$ indicates clear benzoid and quinoid ring vibrations, thereby indicating the oxidation state of ES polyaniline [45]. Several other bands belonging to DNNSA-PANI shown in Fig. (7) are also labeled following Ref. [45].

The characteristics of the Raman spectral features have been identified and detailed quantitative analysis in terms of the highest frequency band position and the intensity ratio of $\mathrm{D}$ to $\mathrm{G}$ band $\left(I_{D} / I_{G}\right)$ is provided in Fig. (8) for all of the spectra. There are strong correlations between the microstructural changes due to nanotubes incorporation that were deduced from the Raman spectral features. Generally speaking, shift (either increase or decrease) in the highest frequency $G$ band is a measure of (a) different $\mathrm{sp}^{2}$ Cconfiguration (since $\mathrm{G}$ band is due to $\mathrm{sp}^{2}$ sites only and refers to $\mathrm{C}-\mathrm{C}$ stretching mode); (b) curvature induced re-hybridization and (probably) mixed hybridized character and (c) compressive or tensile stress/strain, which ranges from $1580 \rightarrow 1605 \mathrm{~cm}^{-1}$ for MWNT and $1592 \rightarrow 1604 \mathrm{~cm}^{-1}$ for SWNT nanocomposites. The position of the D band also changes as shown in insets of Fig. (8a, 8b) and they deserve much needed attention as their variation can be utilized to evaluate mechanical deformations in carbon nanotube-polymer nanocomposites. Qualitatively, the intensity ratio $\left(I_{D} / I_{G}\right)$ is a measure of phase purity (or, degree of disorder). The relative increase of intensity ratio for DNNSA-PANI/SW $(0.1 \rightarrow 0.35)$ as compared to DNNSA-PANI/MW $(0.84 \rightarrow 0.74)$ indicates a concomitant increase in defects and surface functionalization of SWNT with sulfonic group of DNNSA-PANI or possibly others thus reflective of a strong interaction with the host material system. Moreover, it is worthy to note that DNNSA-PANI/NT is not a simple material rather a hybrid electronic structure which is not a simple sum of the individual components. Such materials offer a great promise as sensors because the attached functionality may be tailored for specific chemical or biological analytes, as elucidated in Ref. [46]. This is not only the change in the electronic conductivity and corresponding EDOS, but also some kind of chemical doping due to charge transfer via sulfonic group from the surface of the nanotubes resulting in electrical conductivity variations.

Second-order Raman spectroscopy also provides information about mechanical deformation and the Raman spectra 
(a)

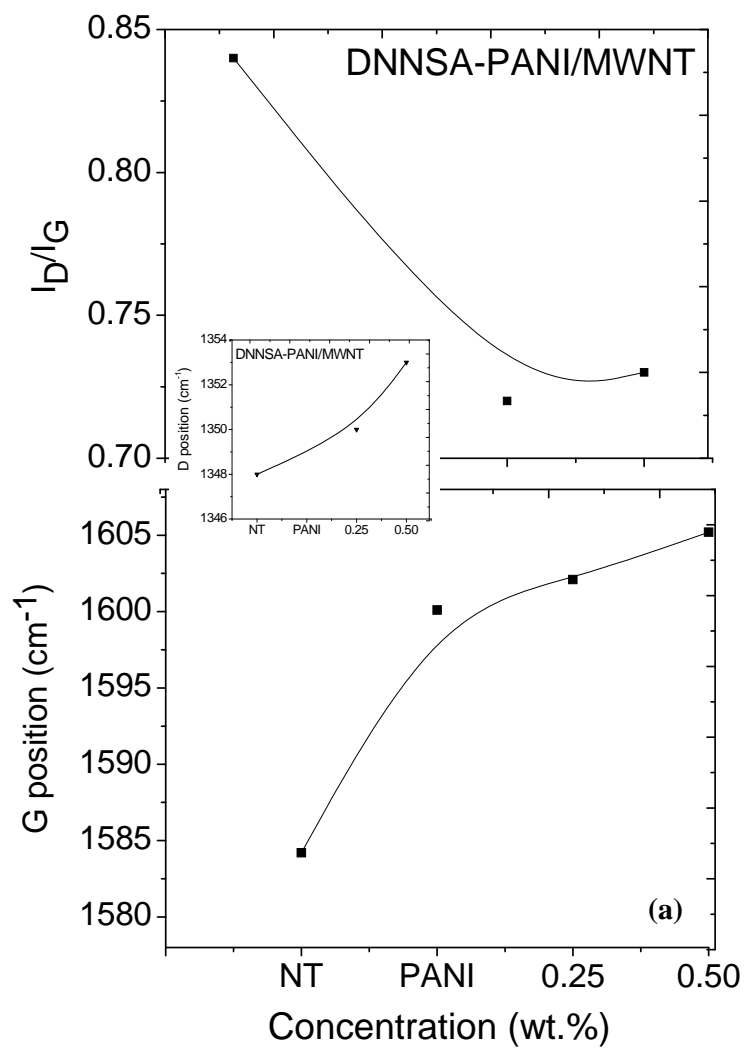

(b)

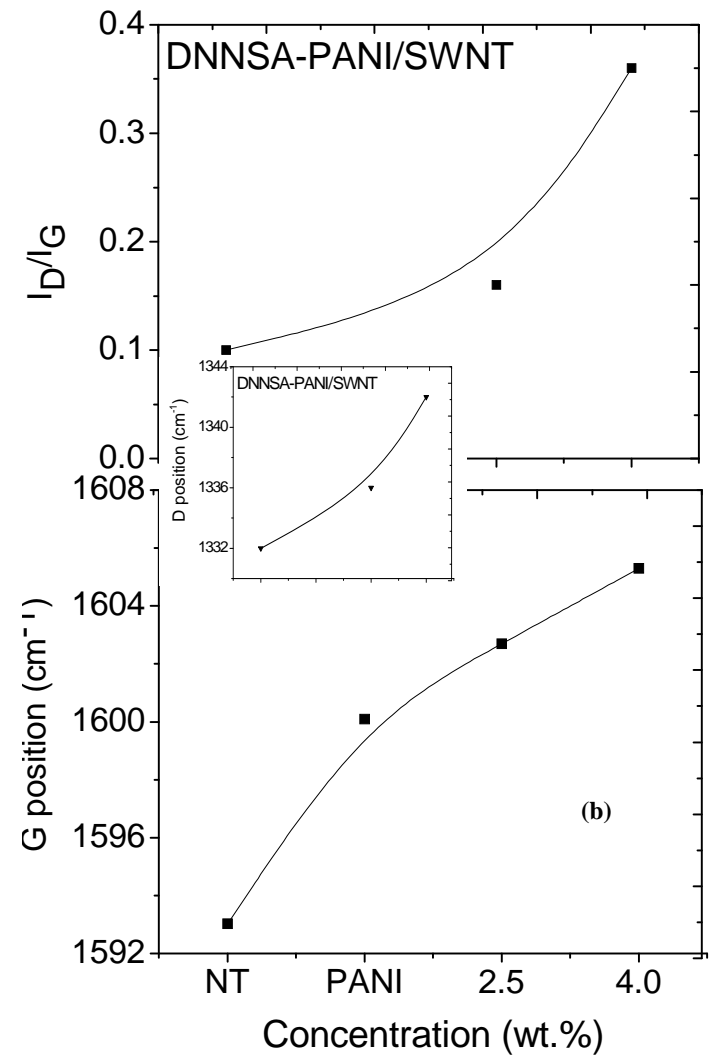

Fig. (8). Shown are the analyses of Raman spectra shown in Fig. (7) in terms of $\mathrm{G}$ band position and the intensity ratio of $\mathrm{D}$ to $\mathrm{G}$ band $\left(\mathrm{I}_{\mathrm{D}} / \mathrm{I}_{\mathrm{G}}\right.$ ) for (a) $\mathrm{MW}$ and (b) $\mathrm{SW}$ nanocomposites. Variation of $\mathrm{D}$ band is also shown in inset. and the variation of second-order D band (i.e. $\mathrm{G}^{*}$ band) at around $2678 \mathrm{~cm}^{-1}$ for both the SWNT and MWNT is shown in Figs. (9a, 9b) and (10), respectively. The combination band (D+G) and second order G band at $\sim 2920 \mathrm{~cm}^{-1}$ and $3200 \mathrm{~cm}^{-1}$, respectively were too weak to be detected [47, 48]. The $G^{*}$ band in the Raman spectra is generally much more intense than the disorder-induced $\mathrm{D}$ band for various $\mathrm{sp}^{2} \mathrm{C}$ materials. It is due to the fact that the $\mathrm{G}^{*}$ band is symmetry allowed by momentum conservation requirements and results from a two-phonon scattering that is explained by double-resonance theory [49], whereas the disorder-induced $\mathrm{D}$ band appears only when there is a breakdown in the inplane translational symmetry. It is used to obtain information on local deformations depending upon its variation in position either towards higher (microscopic compressive) or lower (microscopic tensile) values. To remind ourselves, the $\mathrm{G}^{*}$ band arises from breathing motion of $\mathrm{sp}^{2}$ rings having $\mathrm{A}_{1 \mathrm{~g}}$ symmetry. An upshift implies stiffening of the $\mathrm{C}-\mathrm{C}$ bond length $\left(d_{\mathrm{C}-\mathrm{C}}\right)$, which is a consequence of compressive stress/strain. In view of a linear dependence represented by the following relations through the following equations:

$$
\sigma_{\text {comp }}(G P a)=-0.10 * \Delta \omega\left(\mathrm{cm}^{-1}\right)
$$

and

$$
\frac{\Delta L}{L}=-\frac{\Delta \omega}{\omega}
$$

where $\sigma_{\text {comp }}$ is the microscopic compressive stress, $\mathrm{L}$ is the change in C-C bond distance and $\omega$ is the frequency of $\mathrm{G}^{*}$ band [50-52]. On the basis of Eqn. (1), the estimates of the pressure exerted on NTs varied between -0.4 to $-0.6 \mathrm{GPa}$ for MWNT and SWNT along with polymer matrix, respectively. Since the calculated pressures are not too high, it does not lead to hexagonal distortion as reported in earlier studies [51]. Change in bond length or in-plane lattice constant $\left(d_{\mathrm{C}-}\right.$ $\mathrm{C}, \mathrm{A}^{\circ}$ ) and the corresponding microscopic compressive strain is approximately $-0.17 \times 10^{-3}$ and $-2.2 \times 10^{-3}$ following Eqn. (2) for MWNT and SWNT, respectively.

Fig. (11) displays traditional electrical measurements as I-V plots determining resistivity (or, conductivity) for pristine DNNSA-PANI and both the MWNT and SWNT composite films as a function of nanotube concentration ranging from $0.1 \%$ to $4 \%$ by weight. The inset is an optical micrograph of two finely tipped probes separated by $<0.5 \mathrm{~mm}$ touching the surface of the sample. An electric field is applied, which in turn induces a current flow measured by the Keithley digital multimeter. From these curves (Fig. 11) it can be concluded that DNNSA-PANI/SW samples with the maximum weight fraction has less resistance (steeper curve) than those of DNNSA-PANI/MW and they are in the following order: $(1 / \mathrm{R})_{\text {PANI/SW }}>(1 / \mathrm{R})_{\text {PANI-MW }}>(1 / \mathrm{R})_{\text {PANI }}$. The data in Fig. (12) shows that the logarithmic of $d c$ electrical conductivity $\left(\log _{10} \sigma_{\mathrm{dc}}\right)$ for the DNNSA-PANI and nanocomposite films derived from four-point probe measurements. The conductivity values increases over six orders of magnitude as the nanotube type (MW and SW) and concentration is varied and it can be extrapolated to reported values of SWNT mat values at $100 \%$ loading. Fig. (12) also indicates that the conductivity of the composite starts to saturate for higher SWNT concentration implicating a percolation threshold regime 

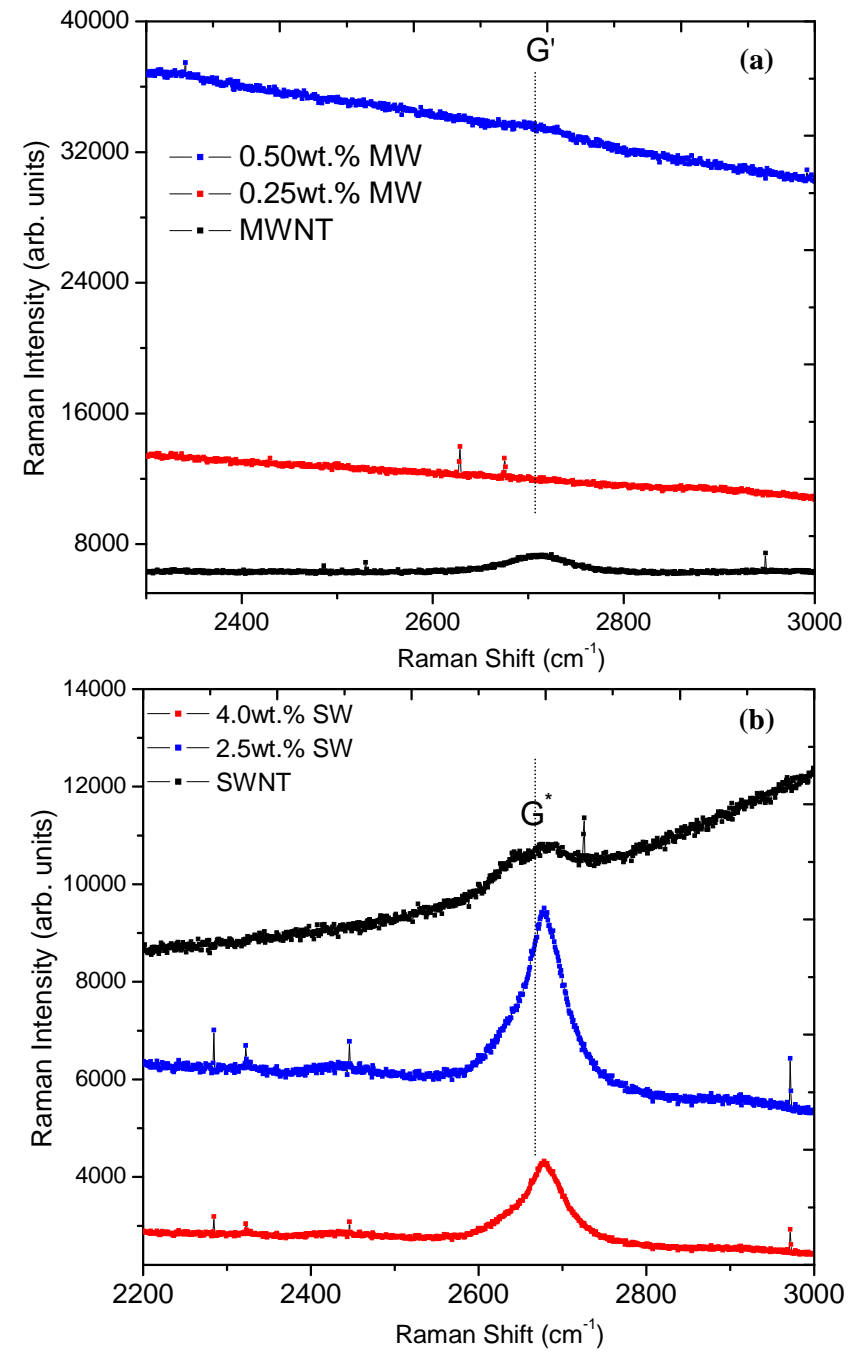

Fig. (9). Shown are the second-order Raman spectra for all of the DNNSA-PANI-NT samples depicting the significant feature including second-order $\mathrm{D}$ band (i.e. $\mathrm{G}^{*}$ ), which is related to mechanical deformation.

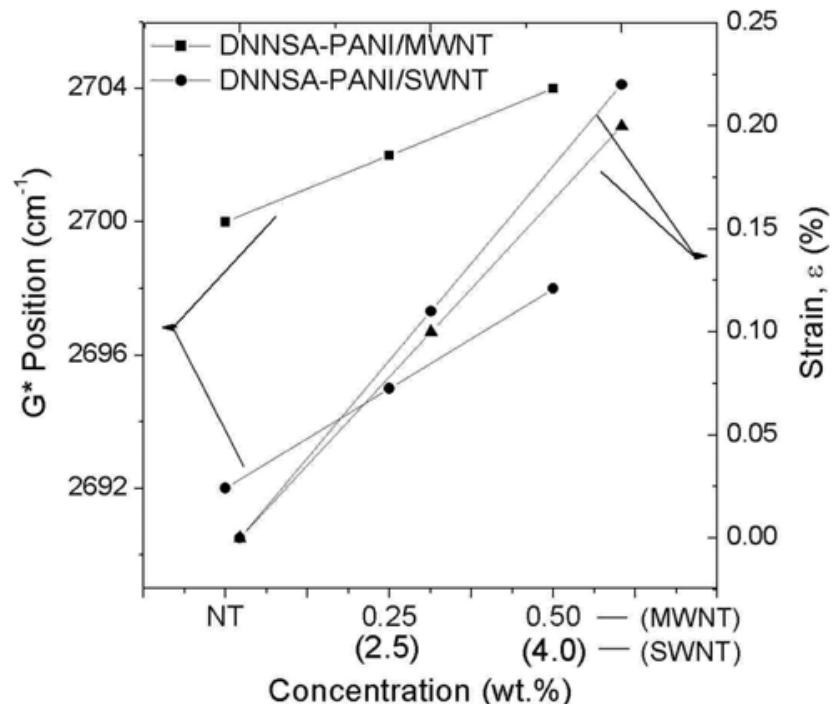

Fig. (10). Shown are the variation of $\mathrm{G}^{*}$ band with concentration of nanotubes by wt.\% in DNNSA-PANI for all of the samples. The calculated strain $(\varepsilon)$ from the position of $\mathrm{G}^{*}$ band is also provided.
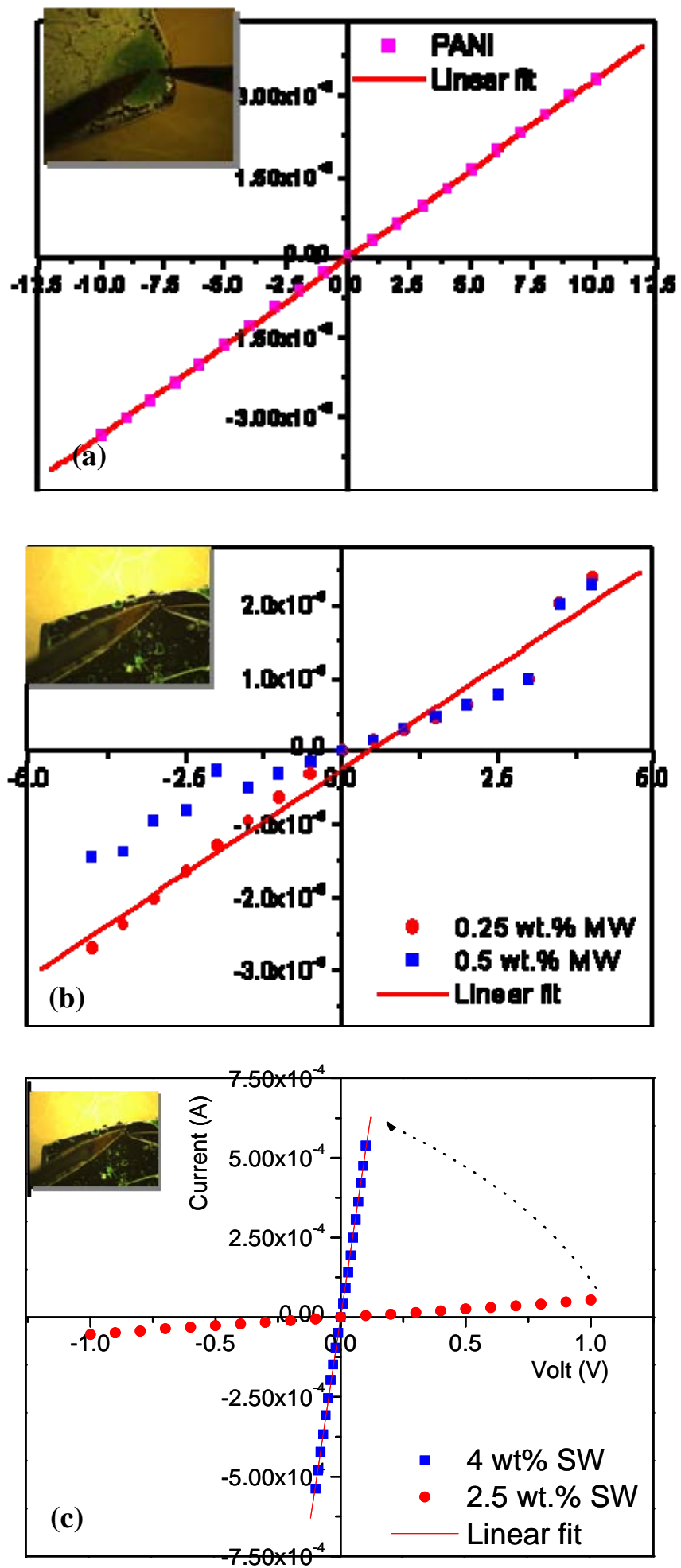

Fig. (11). (a-c) $I-V$ curves provide information on the electrical behavior of all of the samples. Insets show the optical micrographs of the samples along with probes where the data was taken.

$c a$. to 0.5 wt.\% [9] Additionally, the conductivity is highly dependent on the dispersion procedure and possibly independent of the nanotube synthesis method. These results suggest that neither the graphitic component of the nanotube material (higher graphitic content in laser material) nor the metal catalysts associated with the different fabrication methods impact the nanocomposites conductivity. In con- 
trast, the effect dispersion procedure on the composite conductivity reflects difference in contact resistance and charge transport. These results indicate the uniqueness of polyaniline as a conductive binder leading to enhanced properties in DNNSA-PANI/NT composites.

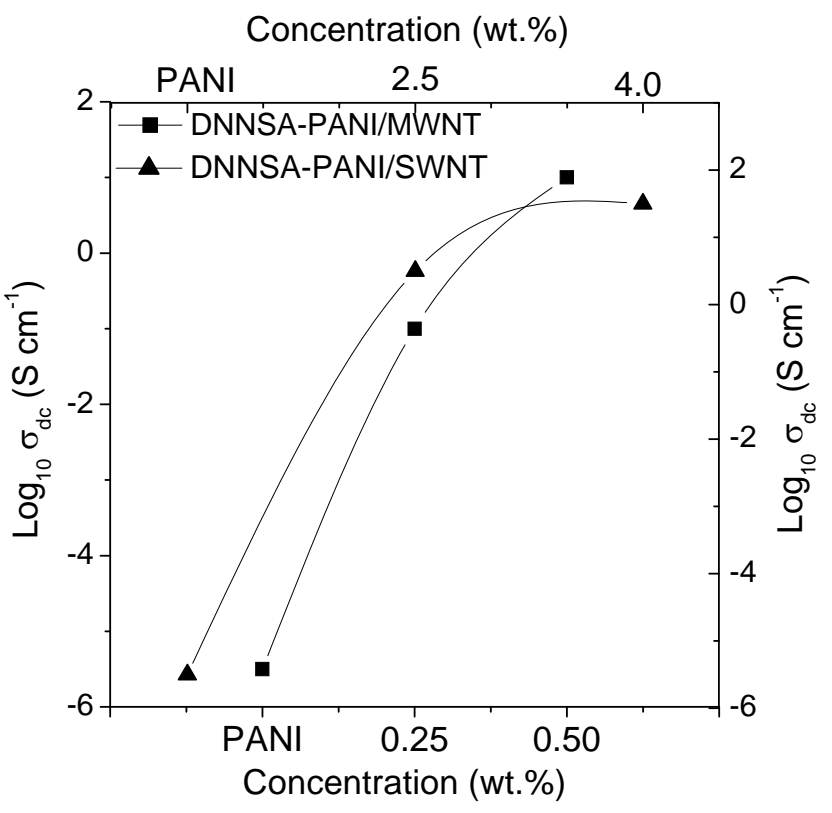

Fig. (12). Shown is the logarithmic plot of $d c$ conductivity in $\mathrm{S} \mathrm{cm}^{-1}$ versus concentration of nanotubes by wt.\% in DNNSA-PANI.

Despite the development of PANI-NT nanocomposites, some of the issues still remain and needs further work include (a) the potential interactions between NTs and conjugated polymers is still intriguing, even though it can be considered that a physical doping of the polymer by NTs is possible by charge transfer as well as $\pi-\pi^{*}$ interactions. Such interactions can increase the electron delocalization which enhances the conductivity of the polymer, (b) there is a great extent of inhomogeneity which can be overcome by functionalizing the nanotubes surface and then there will be a low level of microscopic phase separation/segregation of the components that may lead to higher electrical conductivity. Above all, nanofunctional materials with tailor-made properties can be synthesized from pre-selected constituent molecular building blocks. A possible synthetic route for the preparation of such a nanocomposite material would be in which carbon nanotubes are grafted with conjugated polymers. This may have significant advantage over a composite prepared by simple mixing of components for enhanced physical (electrical, mechanical) properties for a myriad of applications.

\section{CONCLUSION}

In summary, we have prepared and investigated polyaniline-nanotube composites. Scanning electron microscopy revealed the surface morphology which appears to be rough, globular- or cobbled stone-like similar to the ones observed with atomic force microscopy. Homogenous dispersion of nanotubes in the polymer matrix was not as efficient as sought for and showed tangles and spherical agglomerates so-called inhomogeneous distribution. Through XRD structural evaluation, the variation of crystallite size was found to be consistent. Qualitatively, nanotube inclusion was demonstrated using Raman spectroscopy analyses. Although weak, a change in the Raman intensity indicates partial interaction with polymer matrix and debundling. If no such signatures were present, that was indicative of no incorporation. However, it lacked homogenous distribution and support partial interaction, attributed to weaker stiffness of the polymer, lack of strong interfacial interaction and possible departure from the ideal mechanical properties, desired for technological applications. Lastly, the room temperature resistivity measurements showed promising results of an increase in electrical conductivity as a function of nanotube loading that tend to reach maxima for the highest multi-walled nanotube concentration and plateaus for the single-walled nanotubes. These studies also provided a contrasting comparison between single- and multi-walled nanotubes. There is much work needs to be done in terms of processing polymer matrix and nanotubes and role of dopant in polyaniline, which may help to determine and understand the enhanced physical properties for the technological applications sought such as printable organic electronic and sensor devices, electrodes for fuel cell and high energy density Li batteries, space and naval applications.

\section{ACKNOWLEDGEMENTS}

The corresponding author (SG) gratefully acknowledges the SEM and AFM facilities housed in the Center for Applied Science and Engineering (CASE) directed by Dr. R.E. Giedd and operated by Mr. R.J. Patel. We would also like to thank Mr. V. Kandagor for technical assistance who worked as an undergraduate student while the author (SG) was at the Missouri State University, Springfield, MO and finally Prof. R. Hauge (Rice University, TX) for providing HiPCo SWNT used in this work.

\section{REFERENCES}

[1] Iijima S. Helical microtubules of graphitic carbon. Nature 1991; 354: 56-8.

[2] Dresselhaus MS, Dresselhaus G, Avouris P. Carbon Nanotubes: Synthesis, Structure, Properties and Applications, vol. 80, Berlin, Springer, 2001.

[3] Gupta S, Saxena A. Novel nanocarbons: Global topology and curvature perspectives. Mater Res Soc Proc 2006; 981E: N9.6-15.

[4] De Heer WA, Ugarte D. Carbon onions produced by heat treatment of carbon soot and their relation to the $217.5 \mathrm{~nm}$ interstellar absorption feature. Chem Phys Lett 1993; 207: 480-6.

[5] Poncharal P, Wang ZL, Ugarte D, de Heer WA. Electrostatic deflections and electromechanical resonances of carbon nanotubes. Science 1999 ; 283: 1513-6.

[6] Baughman RH, Zakhidov AA, de Heer WA. Carbon Nanotubes-the route toward applications. Science 2002; 297(5582): 787-92.

[7] Philip B, Xie J, Chandrasekhar A, Abraham J, Vardan VK. A novel nanocomposite from multiwalled carbon nanotubes functionalized with a conducting polymer. Smart Mater Struct 2004; 13: 295-8.

[8] Koji M. Biomedical sensors by soft-MEMS techniques. Funct Mater 2005; 25: 34-41.

[9] Blanchet GB, Fincher CR, Gao F. Polyaniline nanotube composites: A high-resolution printable conductor. Appl Phys Lett 2003; 82: 1290-92.

[10] Winey KI, Vaia RA. Introductory article: Polymer Nanocomposites. Mater Res Soc Bull 2007; 32: 314.

[11] Hamon MA, Chen J, Hu H, et al. Dissolution of Single-Walled carbon nanotubes. Adv Mater 1999; 11: 834-40.

[12] Sun YP, Fu K, Lin Y, Huang W. Organic Functionalization of Carbon Nanotubes. J Am Chem Soc 2002; 124: 760-1.

[13] Niyogi S, Hamon MA, Hu H, et al. Chemistry of Single-Walled carbon nanotubes. Acc Chem Res 2002; 35: 1105-13. 
[14] Sun Y, Huang W, Lin Y, et al. Soluble dendron-functionalized carbon nanotubes: Preparation, characterization, and properties. Chem Mater 2001; 13: 2864-69.

[15] Ajayan PM, Stephan O, Collie C, Trauth D. Aligned carbon nanotube arrays formed by cutting a polymer resin-nanotube composite. Science 1994; 265: 1212-14.

[16] Dai L, Mau AWH. Controlled synthesis and modification of carbon nanotubes and C60: carbon nanostructures for advanced polymeric composite materials. Adv Mater 2001; 13: 899-913.

[17] McWilliams A. Nanocomposites, Nanoparticles, Nanoclays, Nanotubes (NANO21C, BCC Research, Norwalk, CT, 2006).

[18] Vaia RA, Giannelis EP. Polymer nanocomposites: Status and opportunities. Mater Res Soc Bull 2001; 26: 394-401.

[19] Vaia RA, Wagner HD. Nanocomposites: issues at the interface. Mater Today 2004; 7: 32-42.

[20] Polymer Nanocomposites Create Exciting Opportunities in the Plastics Industry. Principia Partners, Jersey City, NJ, 2005.

[21] Chen GZ, Shaffer MSP, Coleby D, et al. Carbon nanotube and polypyrrole composites: coating and doping. Adv Mater 2000; 12: 5226.

[22] Ago H, Petritsch K, Shaffer MSP, Windle AH, Friend RH. Composites of carbon nanotubes and conjugated polymers for photovoltaic devices. Adv Mater 1999; 11: 1281-5.

[23] Kim JY, Kim M, Kim HM, Joo J, Choi JH. Electrical and optical studies of organic light emitting devices using SWCNTs-polymer nanocomposites. Opt Mater 2003; 21: 147-51.

[24] Hughes M, Chen GZ, Shaffer MS, Fray DJ, Windle AH. Electrochemical capacitance of a nanoporous composite of carbon nanotubes and polypyrrole. Chem Mater 2002; 14: 1610-13.

[25] Downs C, Nuget J, Ajayan PM, Duquette DJ, Santhanam KSV. Efficient polymerization of aniline at carbon nanotube electrodes. Adv Mater 1999; 11: 1028-31.

[26] Gao M, Huang S, Dai L, Wallace G, Gao R, Wang Z. Aligned coaxial nanowires of carbon nanotubes sheathed with conducting polymers. Angew Chem 2000; 112: 3810-13.

[27] Valter B, Ram MK, Nicolini C. Synthesis of multiwalled carbon nanotubes and poly(o-anisidine) nanocomposite material: fabrication and characterization of its langmuir-schaefer films. Langmuir 2002; 18: 1535-41.

[28] Fan J, Wan M, Zhu D, Chang B, Pan Z, Xie SJ. Synthesis, characterizations, and physical properties of carbon nanotubes coated by conducting polypyrrole. Appl Polym Sci 1999; 74: 2605-10.

[29] Kymakis E, Amaratunga GAJ. Single-wall carbon nanotube/conjugated polymer photovoltaic devices. Appl Phys Lett 2002; 80: 112-4

[30] Woo HS, Czerw R, Webster S, Carroll DL, Park JW, Lee JH. Organic light emitting diodes fabricated with single wall carbon nanotubes dispersed in a hole conducting buffer: the role of carbon nanotubes in a hole conducting polymer. Synth Met 2001; 116: 369-72.

[31] Curran SA, Ajayan PM, Blau WJ, et al. A composite from poly(mphenylenevinylene-co-2,5-dioctoxy-p-phenylenevinylene) and carbon nanotubes: a novel material for molecular optoelectronics. Adv Mater 1998; 10: 1091.

[32] In het Panhuis M, Maiti A, Dalton AB, et al. Selective interaction in a polymer-single-wall carbon nanotube composite. J Phys Chem B 2003; 107: 478-82.

[33] Blanchet GB, Loo YL, Rogers JA, Fincher CR, Gao F. Large area, high resolution, dry printing of conducting polymers for organic electronics. Appl Phys Lett 2003; 82: 463-5.
[34] Heeger AJ, MacDiarmid AG. Transport, magnetic and structural studies of polyacetylene. Mol Cryst Liq Cryst 1981; 77: 1-24.

[35] Chiang JC, MacDiarmid AG. 'Polyaniline': Protonic acid doping of the emeraldine form to the metallic regime. Synth Met 1986; 13 : 193-205.

[36] Yao Y, Smith P, Heeger A. Counter-ion induced processibility of conducting polyaniline and of conducting polyblends of polyaniline in bulk polymers. Synth Met 1992; 48: 91-7.

[37] Zengin G, Shou W, Jin J, et al. Carbon nanotube doped polyaniline. Adv Mater 2002; 14: 1480-83

[38] Kinlen PJ. PANI-DNNSA organic synthesis. US Patent No. 5,863, 465, 1999.

[39] Carver RL, Peng HQ, Sadana AK, et al. a model for nucleation and growth of single wall carbon nanotubes via the hipco process; a catalyst concentration study. J Nanosci Nanotechnol 2005; 5: 103540.

[40] Gill PR, Murray W, Wright MH. The Levenberg-Marquardt Method, Sec. 4.7.3 in Practical Optimization, Academic Press, London, 1981, 136-37.

[41] Ferrari AC, Robertson J. Resonant Raman spectroscopy of disordered, amorphous, and diamond-like carbon. Phys Rev B 2001; 64 : 075414-1-13.

[42] Rao AM, Richter E, Bandow S, et al. Diameter-selective raman scattering from vibrational modes in carbon nanotubes. Science 1987; 275: 187-91.

[43] Blasé X, Benedict LX, Shirley EL, Louie SG. Hybridization effects and metallicity in small radius carbon nanotubes. Phys Rev Lett 1994; 72: 1878-81

[44] Berber S, Kwon YK, Tomanek D. Electronic and structural properties of carbon nanohorns. Phys Rev B 2000; 62: R2291-94.

[45] Furukawa Y, Ueda F, Hyodo Y, Harada I, Nakajima T, Kawagoe T. X-Ray structure of polyaniline. Macromolecules 1994; 24: 77989.

[46] Bekyarova E, Kalinina I, Itkis ME, Leanne Beer L, Cabrera N, Haddon RC. Mechanism of ammonia detection by chemically functionalized single-walled carbon nanotubes: In situ electrical and optical study of gas analyte detection. J Am Chem Soc 2007; 129: 10700-06.

[47] Gupta S, Patel RJ. Changes in the vibrational modes of carbon nanotubes induced by electron-beam irradiation: resonance Raman spectroscopy. J Raman Spectrosc 2007; 38: 188-99.

[48] Gupta S, Kandagor VC, Patel RJ, Ding Y, Hague R. Selfassembled conductive network of carbon nanotubes - polyaniline forming potential nanocomposites. Mater Res Soc Symp Proc 2006; 963E: Q20.1-7.

[49] Saito R, Gruneis A, Samsonidze GG, Brar VW, Dresselhaus G, Dresselhaus MS. Double resonance Raman spectroscopy of singlewall carbon nanotubes. New J Phys 2003; 5: 157.1-157.15.

[50] Wood JR, Zhao Q, Wagner HD. Orientation of carbon nanotubes in polymers and its detection by Raman spectroscopy. Composites Part A: Appl Sci Manuf 2001; 32: 391-99.

[51] Sandler J, Shaffer MSP, Windle AH, et al. Variations in the Raman peak shift as a function of hydrostatic pressure for various carbon nanostructures: A simple geometric effect. Phys Rev B 2003; 67 : $035417-24$

[52] Lourie O, Wagner HD. Evaluation of Young's modulus of carbon nanotubes by micro-Raman spectroscopy. J Mater Res 1998; 13 2418-22. 